\title{
Near-infrared observations of candidate extrinsic S stars
}

\author{
X.-H. Wang ${ }^{1,2}$ and P.-S. Chen ${ }^{1,2}$ \\ 1 Yunnan Observatory, National Observatories, CAS, Kunming 650011, PR China \\ 2 Joint Laboratory for Optical Astronomy, CAS, Beijing 100012, PR China \\ Received 16 May 2001 / Accepted 30 January 2002

\begin{abstract}
Photometric observations in the near infrared for $161 \mathrm{~S}$ stars, including 18 Tc-rich (intrinsic) stars, 19 Tc-deficient (extrinsic) ones and 124 candidates for Tc-deficient S stars, are presented in this paper. Based on some further investigations into the infrared properties of both Tc-rich and Tc-deficient S stars, 104 candidates a convenient way to study the physical properties and the evolutionary status of this species of $\mathrm{S}$ stars.
\end{abstract} \\ are identified as very likely Tc-deficient S stars. The large number of infrared-selected Tc-deficient S stars provides
}

Key words. stars: AGB and post-AGB - stars: evolution - stars: circumstellar matter - infrared: stars

\section{Introduction}

Recent research suggests that two categories of $\mathrm{S}$ stars exist, as proposed early by Iben \& Renzini (1983):

(1) intrinsic S stars, indeed lying on the asymptotic giant branch (AGB) phase of stellar evolution, following the usual $\mathrm{M}-\mathrm{S}-\mathrm{C}$ evolution sequence, and, as a defining characteristic, showing Tc lines in their optical spectra: also called as Tc-rich S stars or S stars with Tc;

(2) extrinsic S stars, populating the first red giant branch (RGB), belonging to white dwarf (WD) containing binary systems, having enhanced $s$-process elements at the stellar surface as the result of mass transfer from an AGB star (now the WD) to a less evolved companion (now the "S star"), but having no Tc: referred to as Tc-deficient $\mathrm{S}$ stars or $\mathrm{S}$ stars without Tc.

The existence of S stars without Tc was first discussed by Scalo \& Miller (1981) on the basis of the data of Little-Marenin \& Little (1979), and has been supported by several further observations (Little et al. 1987; Smith \& Lambert 1988, 1990; Jorissen et al. 1993; and recently Van Eck \& Jorissen 1999). However, it is generally difficult to detect the Tc lines directly since these lines are very weak and lie in a spectral region crowded with many other lines. Of the some $1500 \mathrm{~S}$ stars known in the Galaxy only a tiny minority has been observed for the presence of Tc. Thus we have to find other (indirect) approaches to distinguish Tc-deficient S stars from Tc-rich ones.

Although the periodic radial-velocity variation attributable to the orbital motion of a binary system has been seen for some Tc-deficient stars from Jorissen \& Mayor (1988, 1992), Brown et al. (1990), and more

Send offprint requests to: X.-H. Wang,

e-mail: wang_xunhao@sina.com recently from Udry et al. (1998) and Carquillat et al. (1998), the determination of radial velocity variation is limited by the long orbital periods (on a scale of years). Another approach is to observe the He I $10830 \AA$ triplet in the spectra of Tc-deficient stars (Brown et al. 1990). The He I line is lacking in normal, single giants later than $\mathrm{K}$, and the WD companion is supposed to be an essential prerequisite for the He I line production in Tc-deficient $\mathrm{S}$ stars. Unfortunately, such a prominent feature is undetectable in the vast majority of the spectra of Tc-deficient stars for the triplet almost reaching the cut-off wavelength of the response curve of current CCD cameras. Moreover, excess ultraviolet emission arising from the WD should be visible for Tc-deficient S stars (Johnson et al. 1990, 1993), but the data in the ultraviolet have so far been obtained only from IUE for very few stars. Therefore, it seems that an additional approach, namely studying the infrared colors, may help identify the two categories of S stars for a sample with many stars.

Since the Tc-rich S stars are luminous, cool AGB stars and surrounded by a circumstellar shell, while the Tc-deficient ones are supposed to be less luminous, hot and less evolved, the infrared characteristics of the two classes may be very different (Jura 1986; Groenewegen 1993; Jorissen et al. 1993; Jorissen \& Knapp 1998; Van Eck \& Jorissen 2000). In a previous work (Chen et al. 1998, hereafter Paper I) we studied in detail the infrared properties of both categories of S stars on the basis of our near infrared photometry and the IRAS data for 24 known Tcrich and 20 known Tc-deficient S stars: a series of infrared two-color diagrams were presented, the energy distributions were investigated and the IRAS LRS spectra were discussed.

As work continued from Paper I, we report in this paper the $J H K$ photometric observations for a large sample 
of candidates for Tc-deficient S stars, together with the discussion of some infrared characteristics so as to identify new Tc-deficient S stars.

\section{Sample}

All candidates for Tc-deficient $\mathrm{S}$ stars studied here are selected from $A$ catalogue of associations between IRAS sources and $S$ stars (Chen et al. 1995, hereafter CGJ) on the following criteria:

(1) $V \lesssim 11^{\mathrm{m}} 5$;

(2) $-20^{\circ}<\delta<64^{\circ}$;

(3) the LRS classification is in groups $\mathrm{S}$ or $\mathrm{F}$, or no LRS classification exists; and

(4) $\alpha_{12} \geqq 2.70$, or there is no $\alpha_{12}$ value in the catalog [that means a good quality $25 \mu \mathrm{m}$ flux $($ Flag $>2)$ not available in the IRAS Point Source Catalog (JISWG 1988, hereafter PSC)]. Here, $\alpha_{12}$ is the spectral slope between the 12 and $25 \mu \mathrm{m}$ bands, defined as

$\alpha_{12}=\log \left(25 F_{12} / 12 F_{25}\right) / \log (25 / 12)$.

The $F_{12}$ and $F_{25}$ are fluxes in Jy at 12 and $25 \mu \mathrm{m}$ respectively.

The second criterion is called for by the condition and location of the telescope used. The third one comes from the conclusion of Paper I that LRS classification E is a good indicator of the AGB nature of a star, while candidates for Tc-deficient S stars should be searched for among those in groups $\mathrm{S}$ and $\mathrm{F}$. This claim has also been proposed by Chen \& Kwok (1993) and it is consistent with the analysis of Groenewegen (1993).

Tc-deficient S stars have relatively warm photospheres but have little or no circumstellar material. It should be expected that the fluxes in IRAS bands come from hot stellar photospheres and can thus be represented by the Rayleigh-Jeans tail of the spectra, that is, both spectral indices $\alpha_{12}$ and $\alpha_{23}$ for these stars are near $3\left(\alpha_{23}\right.$ is the 25 to $60 \mu \mathrm{m}$ spectral slope and expressed in a similar form to that of $\alpha_{12}$ ). Out of the S stars whose $\alpha_{12}$ were given by CGJ, about $30 \%$ have $\alpha_{12} \geqq 2.70$. These spectra with a slope close to the Rayleigh-Jeans value are indicative of color temperatures in excess of $2000 \mathrm{~K}$ (IRAS Explanatory Supplement, JISWG 1988). Therefore, we shall focus our discussion in this paper on the sources with $\alpha_{12} \geqq 2.70$ so that the fourth criterion is obtained.

The critical $\alpha_{12}$ of 2.70 can, in fact, be deduced from Table 1 and Fig. 1 . In Table 1 the $\alpha_{12}$ value of the $\mathrm{S}$ stars which are believed to be either Tc-rich or Tc-deficient are listed. All stars studied by Jorissen et al. (1993) are involved and several stars from Groenewegen (1993) are supplemented. The $\alpha_{12}$ value is taken from CGJ or, if not available, computed in this work. The number in General Catalogue of Galactic S Stars (Stephenson 1984, hereafter GCGSS), the IRAS name and the LRS classification are also listed. In Fig. 1 the histograms of $\alpha_{12}$ for Tc-rich and Tc-deficient stars are presented. From Table 1 and
Table 1. $\alpha_{12}$ values of the S stars whose Tc contents are well known.

a. Tc-deficient $\mathrm{S}$ stars.

\begin{tabular}{|c|c|c|c|}
\hline GCGSS & IRAS & $\alpha_{12}$ & LRS \\
\hline 3 & $00051-6235$ & 2.86 & \\
\hline 22 & $01025+1855$ & 2.80 & \\
\hline 26 & $01113+2815$ & 2.83 & $\mathrm{~F}$ \\
\hline 39 & $01309-7913$ & 3.41 & \\
\hline 45 & $01515+2138$ & 3.02 & \\
\hline 79 & $03377+6303$ & 2.81 & $\mathrm{~S}(18)$ \\
\hline 87 & $04030+2435$ & 2.93 & \\
\hline 96 & $04248+2214$ & 3.24 & \\
\hline 104 & $04374-3033$ & 2.80 & \\
\hline 106 & $04470+7955$ & / & \\
\hline 133 & $05199-0842$ & 2.88 & $\mathrm{~S}(16)$ \\
\hline 156 & $05404+0503$ & / & \\
\hline 231 & $06331+1415$ & 2.00 & $\mathrm{~F}(42)$ \\
\hline 233 & $06336+0200$ & 2.36 & \\
\hline 260 & $06457+0535$ & 2.87 & \\
\hline 382 & $07392+1419$ & 2.84 & $\mathrm{~S}(18)$ \\
\hline 494 & $08188+1726$ & 2.94 & I \\
\hline 566 & $08529+0620$ & / & \\
\hline DE Leo & $10226+0902$ & 2.88 & $\mathrm{~S}(01)$ \\
\hline 712 & $10538-1033$ & I & \\
\hline 722 & $11046+6838$ & 2.82 & \\
\hline 829 & $13421-0316$ & / & \\
\hline 926 & $16205+5659$ & 2.80 & \\
\hline 937 & $16418-1359$ & 2.87 & $\mathrm{~S}(19)$ \\
\hline 938 & $16425-1902$ & 2.93 & $\mathrm{~S}(31)$ \\
\hline 981 & $17200+2351$ & 2.72 & \\
\hline 1023 & $18058-3658$ & 2.67 & \\
\hline 1031 & $18128+1614$ & 2.78 & \\
\hline 1092 & $18567+2353$ & / & \\
\hline V1743 Peg & $19323+4909$ & 2.80 & $\mathrm{~S}(17)$ \\
\hline 1173 & $19540-1829$ & / & \\
\hline 1178 & $19581+0113$ & 2.68 & \\
\hline 1192 & $20055+3625$ & 2.90 & \\
\hline 1194 & $20076+3331$ & 2.76 & \\
\hline 1201 & $20152+3124$ & 2.95 & \\
\hline 1209 & $20203+2319$ & 2.67 & \\
\hline 1301 & $22345-1031$ & 2.80 & \\
\hline 1304 & $22415+3339$ & 2.77 & \\
\hline 1322 & $23070+0824$ & 2.90 & $\mathrm{~S}(18)$ \\
\hline 1334 & $23272+2859$ & / & \\
\hline
\end{tabular}

Fig. 1 it is obvious that except for S231 = DY Gem and $\mathrm{S} 233=\mathrm{BD}+2^{\circ} 1307$ which will be discussed in Sect. 4.1, all Tc-deficient $\mathrm{S}$ stars have $\alpha_{12}$ either greater than or very close to 2.70, whereas most Tc-rich ones (74\% of 39) have $\alpha_{12}$ less than 2.70 and the average for this kind of stars is only 2.48 .

Thus, 124 candidates are selected from CGJ.

In addition, 18 known Tc-rich and 19 known Tcdeficient $\mathrm{S}$ stars are also included in our observational list, as most of them were not studied in Paper I. The final sample consists of $161 \mathrm{~S}$ stars which are observed in $J H K$ bands and investigated on their infrared properties. 
Table 1. continued.

b. Tc-rich S stars.

\begin{tabular}{|c|c|c|c|}
\hline GCGSS & IRAS & $\alpha_{12}$ & LRS \\
\hline 8 & $00192-2020$ & 2.73 & $\mathrm{~F}(16)$ \\
\hline 9 & $00213+3817$ & 1.91 & $\mathrm{E}$ \\
\hline 12 & $00435+4758$ & 2.66 & $\mathrm{~S}(01)$ \\
\hline 49 & $02143+4404$ & 2.15 & $\mathrm{E}(22)$ \\
\hline 89 & $04123+2357$ & 2.65 & $\mathrm{~S}$ \\
\hline 103 & $04352+6602$ & 2.69 & $\mathrm{~S}(17)$ \\
\hline 114 & $04497+1410$ & 2.88 & $\mathrm{~S}(18)$ \\
\hline 116 & $04543+4829$ & 2.51 & $\mathrm{~F}$ \\
\hline 149 & $05374+3153$ & 1.87 & $\mathrm{E}(43)$ \\
\hline 307 & $07043+2246$ & 2.44 & $\mathrm{~F}(16)$ \\
\hline 312 & $07095+6853$ & 2.19 & $\mathrm{~F}$ \\
\hline V Gem & $07203+1311$ & 2.40 & $\mathrm{~F}$ \\
\hline 347 & $07245+4605$ & 1.87 & $\mathrm{E}(23)$ \\
\hline 403 & $07462+2351$ & 2.77 & \\
\hline 411 & $07475-1852$ & 2.64 & \\
\hline 416 & $07499-0459$ & 2.61 & \\
\hline 422 & $07507-1129$ & 2.83 & I \\
\hline 589 & $09076+3110$ & 2.13 & $\mathrm{E}(22)$ \\
\hline HR 4647 & $12106-3350$ & 2.52 & $\mathrm{~S}$ \\
\hline 803 & $12417+6121$ & 2.51 & \\
\hline 866 & $14500-4624$ & 2.71 & I \\
\hline 903 & $15492+4837$ & 1.98 & $\mathrm{E}(41)$ \\
\hline S Her & $16496+1501$ & 2.49 & $\mathrm{~S}(17)$ \\
\hline OP Her & $17553+4521$ & 2.57 & $\mathrm{~S}(17)$ \\
\hline 1053 & $18288+3612$ & 2.77 & \\
\hline 1070 & $18395+0646$ & 2.77 & \\
\hline 1099 & $19008+1210$ & 2.61 & $\mathrm{~S}$ \\
\hline 1117 & $19133-1703$ & 2.41 & $\mathrm{~F}$ \\
\hline 1150 & $19354+5005$ & 1.95 & $\mathrm{E}(22)$ \\
\hline 1165 & $19486+3247$ & 2.77 & $\mathrm{E}$ \\
\hline 1188 & $20026+3640$ & 2.28 & $\mathrm{~S}(31)$ \\
\hline 1193 & $20062+0451$ & 2.67 & \\
\hline 1195 & $20100-6225$ & 2.76 & $\mathrm{~S}(18)$ \\
\hline 1198 & $20120+1651$ & 2.50 & \\
\hline 1226 & $20303+1716$ & 2.58 & \\
\hline 1254 & $\mathrm{a}$ & & \\
\hline HR 8062 & $\mathrm{a}$ & & \\
\hline 1292 & $22159-2109$ & 2.34 & $\mathrm{~F}$ \\
\hline 1294 & $22196-4612$ & 2.00 & $\mathrm{E}(42)$ \\
\hline 1315 & $22521+1640$ & 2.84 & $\mathrm{~S}$ \\
\hline 1346 & $23595-1457$ & 2.65 & $\mathrm{~F}(16)$ \\
\hline
\end{tabular}

a In a region of the sky not surveyed by IRAS.

\section{Observation and data reduction}

Near infrared observations were made from Dec. 1995 to Oct. 1997 by using the 1.26-m infrared telescope at the Xinglong Station, Beijing Astronomical Observatory, China. The photometric system and the standard stars used are the same as those described in Paper I. A diaphragm of $16^{\prime \prime}$ was used throughout. For each night the atmospheric extinction coefficients at $J H K$ bands were

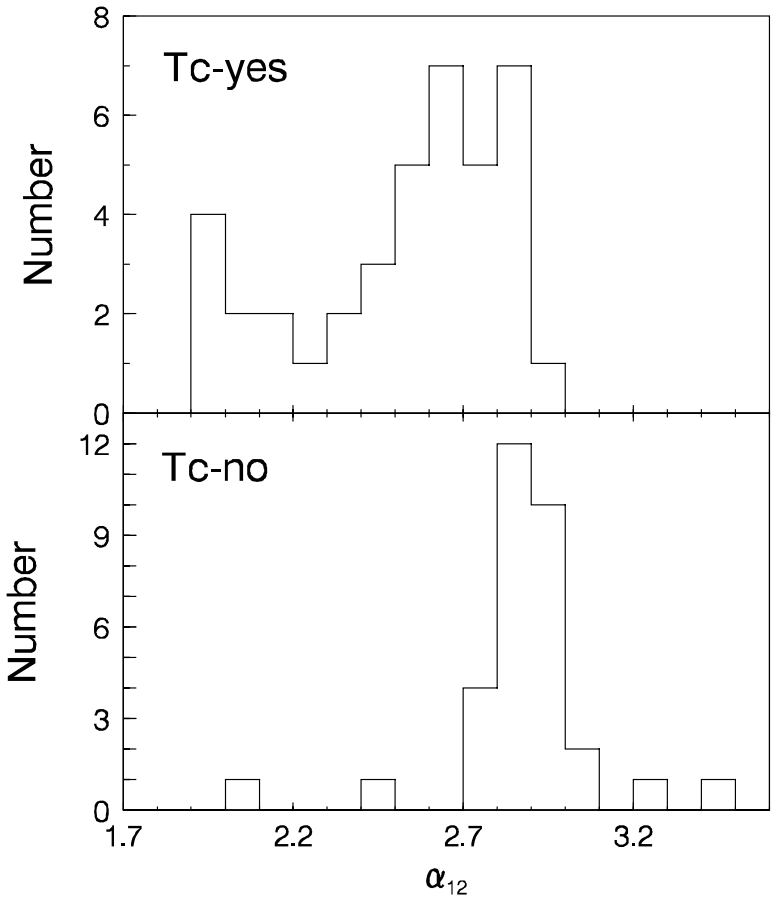

Fig. 1. Histogram of the 12 to $25 \mu \mathrm{m}$ spectral slope for known Tc-rich and Tc-deficient S stars.

obtained by measuring a standard star at different zenith distances. In order to minimize the influence of local changes in atmospheric conditions, most of the observations were made near the zenith and, typically, the airmass difference between the program star and the standard one was less than 0.1. The photometric accuracies derived from the measurements of standard stars are $0.03-0{ }^{\mathrm{m}} 07$.

To estimate the interstellar reddening, van Herk's (1965) interstellar extinction relation

$A_{V}=0.14 \csc |b|[1-\exp (-10 d \sin |b|)]$,

where $b$ is the galactic latitude and $d$ the distance in kpc, and Rieke \& Lebofsky's (1985) reddening law

$A_{J}=0.282 A_{V}, A_{H}=0.175 A_{V}, A_{K}=0.112 A_{V}$

have been used. Although the HIPPARCOS mission has provided trigonometrical parallaxes for $62 \mathrm{~S}$ stars (Van Eck et al. 1998), only 16 stars among them are in common with those observed here, and what is more, only 8 stars have useful parallaxes (i.e., the ratio of the standard error of annual parallax to the parallax itself is between 0 and 1.0). Therefore, an average absolute $K$ magnitude $M_{K}=-8.5$ (Feast et al. 1976) would be assumed for all observed $\mathrm{S}$ stars, from which $d$ and $A_{V}$ may be estimated by means of an iterative method. Then the interstellar reddening was corrected for JHK magnitudes.

The observational results with observing dates are listed respectively in Table 2 for known Tc-deficient and Tc-rich S stars, and in Table 3 for the candidates of Tcdeficient stars. In the tables, the "Name" column lists the GCGSS number (preceded with a capital "S") or the 
Table 2. Observed S stars whose Tc contents have been well known. a. Tc-deficient $\mathrm{S}$ stars.

\begin{tabular}{|c|c|c|c|c|c|c|c|c|c|c|c|c|}
\hline Name & IRAS & Date & $V(B)$ & $J$ & $H$ & $K$ & $F_{12}$ & $F_{25}$ & $F_{60}$ & $F_{100}$ & LRS & $\mathrm{CIO}$ \\
\hline S45 & $01515+2138$ & 951227 & 8.3 & .23 & 4.37 & 4.24 & 1.041 & 0.236 & / & / & & $* * *$ \\
\hline \multirow[t]{2}{*}{ S87 } & $04030+2435$ & 951227 & 8.8 & 4.08 & 3.32 & 3.13 & 3.008 & 0.731 & / & / & & vvK \\
\hline & & 960318 & & 4.79 & 3.72 & 3.19 & & & & & & \\
\hline \multirow[t]{2}{*}{ S96 } & $04248+2214$ & 951227 & 9.6 & 4.41 & 3.69 & 3.44 & 2.537 & 0.491 & / & / & & vHK \\
\hline & & 960318 & & 4.63 & 3.74 & 3.57 & & & & & & \\
\hline \multirow[t]{2}{*}{ S156 } & $05404+0503$ & 951227 & 9.1 & 5.51 & 4.71 & 4.49 & 0.910 & / & / & 4.660 & & JHK \\
\hline & & 960318 & & 5.52 & 4.62 & 4.52 & & & & & & \\
\hline $\mathrm{S} 233$ & $06336+0200$ & 960324 & $(10.5)$ & 4.68 & 4.15 & 4.03 & 0.921 & 0.340 & / & / & & vvv \\
\hline S494 & $08188+1726$ & 960314 & 8.9 & 4.39 & 3.59 & 3.28 & 8.412 & 2.021 & / & / & I & JHK \\
\hline S566 & $08529+0620$ & 960314 & 9.0 & 5.27 & 4.45 & 4.29 & 0.974 & / & / & / & & $* * *$ \\
\hline $\mathrm{S} 712$ & $10538-1033$ & 960318 & $(10.6)$ & 5.85 & 4.90 & 4.69 & 0.878 & / & / & / & & $* * *$ \\
\hline S829 & $13421-0316$ & 960314 & $(9.4)$ & 5.18 & 4.32 & 4.15 & 1.006 & / & / & / & & $* * *$ \\
\hline S926 & $16205+5659$ & 960314 & 6.6 & 4.18 & 3.48 & 3.34 & 2.378 & 0.635 & / & / & & $* * *$ \\
\hline S981 & $17200+2351$ & 960314 & 9.4 & 5.53 & 4.90 & 4.65 & 0.688 & 0.195 & / & / & & $* * *$ \\
\hline S1031 & $18128+1614$ & 960324 & 7.4 & 3.22 & 2.49 & 2.30 & 5.591 & 1.514 & / & / & & $* * \mathrm{~K}$ \\
\hline S1092 & $18567+2353$ & 960318 & 9.9 & 5.96 & 5.52 & 5.01 & 0.542 & / & / & / & & $* * *$ \\
\hline S1173 & $19540-1829$ & 971023 & $(10.1)$ & 4.86 & 4.24 & 3.98 & 1.274 & / & / & / & & $* * *$ \\
\hline S1201 & $20152+3124$ & 960324 & 8.6 & 3.70 & 2.99 & 2.82 & 2.876 & 0.687 & / & 22.89 & & $* * *$ \\
\hline S1209 & $20203+2319$ & 971023 & 9.4 & 4.88 & 4.24 & 3.96 & 1.048 & 0.307 & / & / & & $* * *$ \\
\hline S1334 & $23272+2859$ & 951227 & 9.3 & 6.26 & 5.39 & 5.24 & 0.507 & / & / & / & & $* * *$ \\
\hline DE Leo & $10226+0902$ & 960314 & $(5.6)$ & 2.03 & 1.53 & 1.29 & 14.29 & 3.583 & 0.794 & 0.755 & $\mathrm{~S}(01)$ & $\mathrm{v} * \mathrm{~K}$ \\
\hline V1743 Peg & $19323+4909$ & 960324 & $(5.9)$ & 1.62 & 0.87 & 0.72 & 26.02 & 6.946 & 1.022 & / & $\mathrm{S}(17)$ & $\mathrm{J} * \mathrm{~K}$ \\
\hline
\end{tabular}

b. Tc-rich S stars.

\begin{tabular}{lllllllllllll}
\hline Name & IRAS & Date & $V(B)$ & $J$ & $H$ & $K$ & $F_{12}$ & $F_{25}$ & $F_{60}$ & $F_{100}$ & LRS & CIO \\
\hline S9 & $00213+3817$ & 971021 & 6.8 & 2.66 & 1.29 & 0.65 & 327.0 & 167.7 & 24.16 & 10.37 & $\mathrm{E}$ & vvv \\
S49 & $02143+4404$ & 971021 & 10.5 & 1.22 & 0.47 & 0.00 & 167.4 & 72.14 & 13.45 & 5.606 & $\mathrm{E}(22)$ & vvv \\
S89 & $04123+2357$ & 951227 & 6.9 & 2.07 & 1.23 & 0.95 & 24.43 & 7.298 & 2.631 & 2.608 & $\mathrm{~S}$ & $* * \mathrm{~K}$ \\
S103 & $04352+6602$ & 970102 & 8.2 & 1.90 & 1.25 & 1.04 & 41.29 & 11.91 & 3.632 & 2.577 & $\mathrm{~S}(17)$ & Jvv \\
S114 & $04497+1410$ & 951227 & 4.8 & 0.44 & -0.30 & -0.54 & 85.35 & 21.43 & 4.569 & 3.788 & $\mathrm{~S}(18)$ & $* * \mathrm{~K}$ \\
S416 & $07499-0459$ & 960324 & $(10.5)$ & 4.36 & 3.67 & 3.43 & 1.905 & 0.584 & $/$ & $/$ & & $* * *$ \\
S589 & $09076+3110$ & 960314 & 7.0 & -0.58 & -1.33 & -1.62 & 479.9 & 208.7 & 32.58 & 10.10 & $\mathrm{E}(22)$ & $\mathrm{JHK}$ \\
S903 & $15492+4837$ & 960314 & 7.2 & 0.49 & -0.18 & -0.61 & 199.3 & 97.09 & 16.70 & 5.973 & $\mathrm{E}(41)$ & $* * \mathrm{~K}$ \\
S1099 & $19008+1210$ & 960324 & 8.5 & 2.75 & 2.15 & 2.01 & 10.95 & 3.360 & 0.808 & $/$ & $\mathrm{S}$ & vHK \\
S1117 & $19133-1703$ & 971023 & 11.5 & 1.87 & 1.21 & 0.87 & 40.29 & 14.30 & 4.043 & 2.845 & $\mathrm{~F}$ & vvv \\
S1193 & $20062+0451$ & 971023 & 9.1 & 4.63 & 3.90 & 3.67 & 1.781 & 0.522 & 0.547 & $/$ & & $* * *$ \\
S1198 & $20120+1651$ & 971023 & 9.1 & 3.34 & 2.66 & 2.58 & 6.196 & 2.067 & $/$ & $/$ & & $* * \mathrm{~K}$ \\
S1346 & $23595-1457$ & 970102 & $(12.4)$ & 2.77 & 2.36 & 2.22 & 13.31 & 3.964 & 0.725 & $/$ & $\mathrm{F}(16)$ & vvv \\
V Gem & $07203+1311$ & 960324 & 12.2 & 3.32 & 2.63 & 2.48 & 9.241 & 3.313 & 0.362 & $/$ & $\mathrm{F}$ & vvv \\
S Her & $16496+1501$ & 960324 & 8.7 & 2.59 & 1.88 & 1.55 & 34.32 & 11.46 & 1.916 & 0.780 & $\mathrm{~S}(17)$ & Jvv \\
OP Her & $17553+4521$ & 960324 & $(6.4)$ & 1.29 & 0.39 & 0.28 & 54.13 & 17.12 & 3.349 & 2.317 & $\mathrm{~S}(17)$ & $\mathrm{J} * \mathrm{~K}$ \\
S1254 & $\mathrm{a}$ & 971023 & 7.1 & 2.01 & 1.38 & 1.07 & & & & & & $* * \mathrm{~K}$ \\
HR 8062 & $\mathrm{a}$ & 971023 & 5.8 & 1.58 & 1.02 & 0.72 & & & & & & $* * \mathrm{~K}$ \\
\hline
\end{tabular}

${ }^{\text {a }}$ In a region of the sky not surveyed by IRAS.

number in the Stephenson's (1990) catalog for new and relatively faint $\mathrm{S}$ stars (preceded with "Sf") or the variable star name or the HR number. The IRAS associations are taken from CGJ for the great majority of stars and from Jorissen et al. (1993) for several stars not included in GCGSS and, in the case of V Gem, from the IRAS LRS Letter Classification Lists (Kwok et al. 1997, hereafter LRSLC). The IRAS fluxes (in Jy) at 12, 25, 60 and $100 \mu \mathrm{m}$ bands are from PSC and only good quality ones are listed. From LRSLC and the IRAS Atlas of Low-Resolution Spectra (JISWG 1986) both the LRS classification in letter codes defined by Volk \& Cohen (1989) and that in the usual two-digit codes are presented. In the forth column of Tables 2 and 3, the $V(B)$ magnitudes taken from the Hubble Space Telescope Guide Star Catalog (1992) or the GCGSS are given for most stars, but the I magnitudes from Stephenson (1990) are given for his faint $\mathrm{S}$ stars. In the last column (referred to as "CIO") whether near infrared observations appear in the Catalog of Infrared Observations (Gezari et al. 1993, 
Table 3. Observed candidates for Tc-deficient S stars.

\begin{tabular}{|c|c|c|c|c|c|c|c|c|c|c|c|c|c|}
\hline Name & IRAS & Date & $V(B)$ & $J$ & $H$ & $K$ & $F_{12}$ & $F_{25}$ & $F_{60}$ & $F_{100}$ & LRS & $\mathrm{Tc}$ & $\mathrm{CIO}$ \\
\hline S10 & $00300+6342$ & 951227 & 11.6 & 5.09 & 4.69 & 4.47 & 0.657 & / & / & / & & $\mathrm{n}:$ & $* * *$ \\
\hline S32 & $01229+2108$ & 970102 & 10.4 & 6.25 & 5.55 & 5.18 & 0.770 & / & / & / & & & $* * *$ \\
\hline S42 & $01441+5127$ & 951227 & 11.3 & 6.48 & 5.83 & 5.58 & 0.295 & / & / & / & & $\mathrm{n}:$ & $* * *$ \\
\hline S47 & $02048+6208$ & 951227 & 11.6 & 5.27 & 4.90 & 4.86 & 0.449 & / & l & / & & $\mathrm{n}:$ & $* * *$ \\
\hline S52 & $02151+3130$ & 951227 & 9.1 & 5.42 & 4.71 & 4.43 & 1.670 & 0.454 & / & / & & & vvv \\
\hline S57 & $02228+3753$ & 951227 & 10.2 & 3.04 & 2.35 & 2.08 & 10.27 & 2.807 & 0.643 & 0.964 & $\mathrm{~S}(01)$ & $\mathrm{n}$ & $* * \mathrm{~K}$ \\
\hline \multirow[t]{2}{*}{ S76 } & $03327+6403$ & 960318 & 12.0 & 5.81 & 4.70 & 4.48 & 0.992 & / & / & / & & $\mathrm{n}:$ & $* * *$ \\
\hline & & 970101 & & 5.84 & 4.72 & 4.54 & & & & & & & \\
\hline S78 & $03361+5056$ & 951227 & 10.5 & 4.18 & 3.56 & 3.37 & 2.217 & 0.599 & / & / & & $\mathrm{n}$ & $* * *$ \\
\hline \multirow[t]{2}{*}{ S 80} & $03406+2216$ & 951227 & 9.1 & 4.86 & 4.06 & 3.87 & 1.645 & 0.423 & / & / & & $\mathrm{n}$ & $* * *$ \\
\hline & & 960318 & & 4.86 & 4.05 & 3.77 & & & & & & & \\
\hline $\mathrm{S} 81$ & $03433+5254$ & 951227 & 10.2 & 3.75 & 3.00 & 2.88 & 3.915 & 0.983 & / & / & & $\mathrm{n}$ & $* * \mathrm{~K}$ \\
\hline S83 & $03482+0645$ & 951227 & 10.6 & 6.44 & 5.58 & 5.27 & 0.526 & / & / & / & & $\mathrm{n}:$ & $* * *$ \\
\hline S 86 & $03572+6133$ & 951227 & 11.0 & 5.97 & 5.14 & 4.95 & 0.691 & / & / & / & & $\mathrm{n}:$ & $* * *$ \\
\hline \multirow[t]{2}{*}{ S94 } & $04218-0238$ & 951227 & $(10.5)$ & 5.51 & 4.92 & 4.65 & 0.831 & 0.211 & / & 0.675 & & $\mathrm{n}$ & vvv \\
\hline & & 960324 & & 5.54 & 4.72 & 4.57 & & & & & & & \\
\hline S101 & $04323+4040$ & 951227 & 11.6 & 6.46 & 5.56 & 5.41 & 0.378 & / & l & / & & $\mathrm{n}:$ & $* * *$ \\
\hline S102 & $04328+1235$ & 951227 & 10.0 & 3.81 & 3.13 & 2.68 & 5.394 & 1.534 & / & / & & $\mathrm{n}$ & JHK \\
\hline S105 & $04414+4616$ & 951226 & 9.7 & 5.27 & 5.31 & 5.30 & 0.390 & / & / & / & & $\mathrm{n}:$ & $* * *$ \\
\hline S111 & $04484+3958$ & 951227 & 9.7 & 5.14 & 4.78 & 4.58 & 0.681 & / & / & / & & $\mathrm{n}:$ & $* * *$ \\
\hline S115 & $04506+3159$ & 951227 & 11.4 & 6.21 & 5.48 & 5.23 & 0.463 & / & / & / & & $\mathrm{n}:$ & $* * *$ \\
\hline $\mathrm{S} 124$ & $05087+4649$ & 951227 & 10.9 & 5.67 & 5.01 & 4.84 & 0.631 & / & / & / & & $\mathrm{n}:$ & $* * *$ \\
\hline S132 & $05190+4831$ & 951227 & 11.4 & 5.61 & 4.93 & 4.61 & 0.731 & / & / & / & & $\mathrm{n}:$ & $* * *$ \\
\hline $\mathrm{S} 138$ & $05225+3221$ & 951227 & 11.1 & 5.90 & 5.43 & 5.45 & 0.293 & / & l & / & & $\mathrm{n}:$ & $* * *$ \\
\hline S142 & $05289+4056$ & 951227 & 12.2 & 5.72 & 5.36 & 5.03 & 0.533 & / & / & / & & $\mathrm{n}:$ & $* * *$ \\
\hline \multirow[t]{2}{*}{ S152 } & $05389+5211$ & 951227 & 8.8 & 4.85 & 4.01 & 3.85 & 2.134 & 0.529 & l & / & & $\mathrm{n}$ & $* * *$ \\
\hline & & 960324 & & 4.95 & 4.04 & 3.82 & & & & & & & \\
\hline S153 & $05390+1831$ & 960318 & 8.9 & 3.67 & 2.76 & 2.58 & 5.939 & 1.674 & l & / & & $\mathrm{n}$ & JHK \\
\hline \multirow[t]{2}{*}{ S158 } & $05411+0736$ & 951227 & 9.7 & 4.91 & 4.15 & 4.11 & 1.477 & 0.361 & / & / & & $\mathrm{n}$ & $* * *$ \\
\hline & & 960318 & & 4.73 & 4.05 & 3.88 & & & & & & & \\
\hline S161 & $05446+0239$ & 951227 & 10.8 & 5.65 & 4.98 & 4.67 & 0.790 & / & 0.6 & / & & $\mathrm{n}:$ & $* * *$ \\
\hline S162 & $05449-0423$ & 960318 & $(12.4)$ & 5.47 & 4.43 & 4.26 & 1.044 & 0.286 & / & / & & $\mathrm{n}$ & $* * *$ \\
\hline S163 & $05465+0641$ & 951227 & 10.8 & 6.01 & 5.17 & 4.92 & 0.795 & / & / & / & & & $* * *$ \\
\hline S165 & $05492+2549$ & 951227 & 10.3 & 3.70 & 3.07 & 2.88 & 3.591 & 0.934 & / & / & & $\mathrm{n}$ & $* * *$ \\
\hline S167 & $05484-1708$ & 960318 & $(9.9)$ & 4.79 & 4.13 & 4.03 & 1.160 & 0.272 & / & / & & $\mathrm{n}$ & $* * *$ \\
\hline S171 & $05519+4618$ & 951227 & 11.0 & 6.75 & 5.52 & 5.18 & 0.354 & / & / & / & & $\mathrm{n}:$ & $* * *$ \\
\hline S176 & $05567-1052$ & 960318 & (11.4) & 3.32 & 2.40 & 2.24 & 6.706 & 1.734 & 0.416 & / & & $\mathrm{n}$ & $* * \mathrm{~K}$ \\
\hline S181 & $06001+3138$ & 960318 & 9.6 & 4.17 & 3.67 & 3.19 & 2.659 & 0.754 & / & / & & $\mathrm{n}$ & vvv \\
\hline S183 & $06018+2746$ & 951227 & 11.3 & 5.46 & 5.18 & 4.95 & 0.619 & / & l & / & & $\mathrm{n}:$ & $* * *$ \\
\hline S188 & $06052+2807$ & 951227 & 11.3 & 6.15 & 5.63 & 5.47 & 0.290 & / & / & / & & $\mathrm{n}:$ & $* * *$ \\
\hline S198 & $06105+0127$ & 951227 & $(11.6)$ & 5.99 & 5.30 & 5.12 & 0.450 & / & / & / & & $\mathrm{n}:$ & $* * *$ \\
\hline S207 & $06146+0200$ & 951227 & (11.8) & 5.17 & 4.79 & 4.45 & 1.047 & / & / & / & & $\mathrm{n}:$ & $* * *$ \\
\hline S212 & $06197+0327$ & 960324 & 8.1 & 2.67 & 1.89 & 1.51 & 16.97 & 4.732 & 1.646 & 3.407 & $\mathrm{~S}(18)$ & $\mathrm{n}$ & vvv \\
\hline $\mathrm{S} 213$ & $06197-0751$ & 960324 & $(13.2)$ & 5.62 & 4.98 & 4.65 & 0.856 & 0.193 & / & / & & $\mathrm{n}$ & $* * *$ \\
\hline S214 & $06208+2120$ & 951227 & 11.0 & 5.35 & 4.67 & 4.50 & 0.807 & / & / & / & & $\mathrm{n}:$ & $* * *$ \\
\hline \multirow[t]{2}{*}{ S219 } & $06241+1555$ & 951227 & 9.2 & 4.12 & 3.70 & 3.27 & 2.277 & 0.629 & / & / & & $\mathrm{n}$ & vHK \\
\hline & & 960324 & & 4.02 & 3.44 & 3.32 & & & & & & & \\
\hline S223 & $06298-1338$ & 970315 & (12.4) & 6.06 & 5.23 & 4.98 & 0.517 & / & l & / & & $\mathrm{n}:$ & $* * *$ \\
\hline S227 & $06325+5227$ & 960318 & 10.3 & 5.47 & 4.69 & 4.45 & 0.915 & / & / & / & & $\mathrm{n}:$ & $* * *$ \\
\hline S228 & $06322+3925$ & 960318 & 10.7 & 6.18 & 5.52 & 5.31 & 0.410 & / & l & / & & $\mathrm{n}:$ & $* * *$ \\
\hline S239 & $06368+2541$ & 951227 & 10.9 & 6.21 & 5.61 & 5.38 & 0.302 & / & / & / & & $\mathrm{n}:$ & $* * *$ \\
\hline S247 & $06402+2548$ & 951227 & 8.5 & 5.53 & 4.95 & 4.86 & 0.650 & / & / & / & & $\mathrm{n}:$ & vvv \\
\hline S262 & $06462+0659$ & 960324 & 11.5 & 4.92 & 4.27 & 4.12 & 1.025 & / & / & / & & $\mathrm{n}:$ & $* * *$ \\
\hline S266 & $06477+2905$ & 951227 & 11.4 & 6.96 & 6.11 & 5.91 & 0.728 & / & / & / & & & $* * *$ \\
\hline $\mathrm{S} 270$ & $06509+2612$ & 951227 & 10.4 & 5.88 & 5.02 & 4.42 & 1.428 & 1 & 1 & / & & & $* * *$ \\
\hline
\end{tabular}

hereafter CIO) is reflected by a set of 3 symbols for $J, H$ and $K$ bands respectively. The symbol "*" denotes that there is no observation appearing in CIO catalog, a "J" or an "H" or a " $\mathrm{K}$ " indicates the CIO magnitudes at the corresponding band do not differ more than 0.2 from the value here, and a "v" shows a difference of more than 0.2 existing between the present measurement and one of the previous data in CIO for a corresponding band. Moreover, the column labelled "Tc" in Table 3 stands for the judgment on a star as a Tc-deficient $\mathrm{S}$ star according to the discussion in Sect. 4: a notation " $n$ " means the star should be Tc-deficient as judged by the $(K-[12])-([12]-[25])$ 
Table 3. continued.

\begin{tabular}{|c|c|c|c|c|c|c|c|c|c|c|c|c|c|}
\hline Name & IRAS & Date & $V(B)$ & $J$ & $H$ & $K$ & $F_{12}$ & $F_{25}$ & $F_{60}$ & $F_{100}$ & LRS & $\mathrm{Tc}$ & $\mathrm{CIO}$ \\
\hline $\mathrm{S} 288$ & $06573-1412$ & 970102 & $(11.2)$ & 4.11 & 3.77 & 3.69 & 1.552 & 0.360 & l & / & & $\mathrm{n}$ & $* * *$ \\
\hline S 290 & $06583+1929$ & 960318 & 10.7 & 6.46 & 5.54 & 5.40 & 0.667 & / & / & / & & & $* * *$ \\
\hline S306 & $07037-0346$ & 960324 & (11.6) & 4.40 & 4.14 & 3.76 & 1.427 & 0.372 & / & / & & $\mathrm{n}$ & $* * *$ \\
\hline S311 & $07062+0314$ & 960324 & 9.3 & 5.65 & 5.08 & 4.82 & 0.505 & / & / & / & & $\mathrm{n}:$ & $* * *$ \\
\hline S322 & $07116-0834$ & 970101 & $(11.7)$ & 5.12 & 4.65 & 4.70 & 0.420 & / & / & / & & $\mathrm{n}:$ & $* * *$ \\
\hline S332 & $07165-1109$ & 970315 & $(10.5)$ & 3.39 & 2.97 & 2.74 & 3.452 & 0.804 & / & / & & $\mathrm{n}$ & $* * *$ \\
\hline S334 & $07168-0037$ & 960324 & (12.8) & 5.12 & 4.40 & 4.12 & 2.308 & 0.638 & / & / & & & $* * *$ \\
\hline \multirow[t]{2}{*}{ S335 } & $07169-1122$ & 970101 & $(9.6)$ & 3.25 & 2.60 & 2.48 & 4.293 & 1.182 & / & / & & $\mathrm{n}$ & $* * \mathrm{v}$ \\
\hline & & 970315 & & 3.27 & 2.57 & 2.36 & & & & & & & \\
\hline S 362 & $07296-0924$ & 960318 & $(13.5)$ & 4.74 & 4.00 & 3.93 & 1.405 & 0.348 & / & / & & $\mathrm{n}$ & $* * *$ \\
\hline S363 & $07303+0153$ & 960324 & $(12.7)$ & 6.37 & 5.63 & 5.45 & 0.289 & / & / & / & & $\mathrm{n}:$ & $* * *$ \\
\hline S379 & $07363-1218$ & 960324 & $(13.0)$ & 5.75 & 5.33 & 5.11 & 0.358 & / & / & / & & $\mathrm{n}:$ & $* * *$ \\
\hline S413 & $07496+3444$ & 960324 & 10.2 & 4.90 & 4.06 & 3.77 & 1.873 & 0.507 & / & / & & $\mathrm{n}$ & $* * *$ \\
\hline S418 & $07505+1754$ & 960324 & 8.2 & 4.29 & 3.73 & 3.36 & 2.169 & 0.499 & / & / & & $\mathrm{n}$ & $* * *$ \\
\hline S454 & $08021-0255$ & 960314 & $(12.2)$ & 5.57 & 5.00 & 4.84 & 0.592 & 0.131 & / & / & & $\mathrm{n}$ & $* * *$ \\
\hline S471 & $08083+0817$ & 960314 & 10.6 & 5.21 & 4.44 & 4.20 & 1.112 & 0.308 & / & / & & $\mathrm{n}$ & $* * *$ \\
\hline S836 & $13494-0313$ & 960314 & $(10.6)$ & 4.96 & 4.44 & 4.28 & 0.866 & / & / & / & & $\mathrm{n}:$ & $* * *$ \\
\hline S 855 & $14251-0251$ & 960314 & $(10.5)$ & 5.56 & 4.77 & 4.56 & 0.793 & / & / & / & & $\mathrm{n}:$ & $* * *$ \\
\hline S908 & $15592-1109$ & 960318 & $(10.5)$ & 4.50 & 3.73 & 3.63 & 2.047 & 0.560 & / & / & & $\mathrm{n}$ & $* * *$ \\
\hline S959 & $17047-1303$ & 960314 & $(11.5)$ & 5.69 & 4.24 & 3.62 & 1.926 & / & / & / & & $\mathrm{n}:$ & $* * *$ \\
\hline S964 & $17112+0551$ & 960314 & 8.1 & 4.58 & 3.63 & 3.36 & 2.256 & 0.538 & / & / & & $\mathrm{n}$ & $* * \mathrm{v}$ \\
\hline S968 & $17154-0510$ & 960318 & $(12.7)$ & 6.13 & 5.33 & 4.98 & 0.506 & / & / & / & & $\mathrm{n}:$ & $* * *$ \\
\hline S996 & $17379-0250$ & 960318 & $(9.1)$ & 3.75 & 2.88 & 2.71 & 3.673 & 0.941 & / & / & & $\mathrm{n}$ & $* * \mathrm{~K}$ \\
\hline S1012 & $17563-1438$ & 960318 & 10.9 & 5.32 & 4.34 & 4.18 & 0.856 & / & / & / & & $\mathrm{n}:$ & $* * *$ \\
\hline S1024 & $18052+0027$ & 960318 & 10.8 & 6.43 & 5.54 & 5.51 & 0.436 & / & / & / & & $\mathrm{n}:$ & $* * *$ \\
\hline $\mathrm{S} 1028$ & $18087+2133$ & 960318 & 9.1 & 6.27 & 5.34 & 5.11 & 0.391 & / & / & / & & $\mathrm{n}:$ & $* * *$ \\
\hline $\mathrm{S} 1075$ & $18439+2645$ & 960318 & 8.9 & 6.66 & 5.70 & 5.60 & 0.318 & / & / & / & & $\mathrm{n}:$ & $* * *$ \\
\hline S1083 & $18508-1415$ & 960318 & 11.1 & 5.66 & 4.86 & 4.65 & 1.369 & / & / & / & & & $* * *$ \\
\hline S1097 & $18581+2636$ & 960318 & 11.8 & 6.46 & 5.64 & 5.42 & 0.390 & / & / & / & & $\mathrm{n}:$ & $* * *$ \\
\hline S1105 & $19060+0225$ & 960318 & 10.9 & 5.35 & 4.67 & 4.43 & 0.794 & / & / & / & & $\mathrm{n}:$ & ** \\
\hline $\mathrm{S} 1118$ & $19133+0032$ & 960324 & 11.0 & 4.06 & 3.62 & 3.17 & 2.949 & 0.830 & / & / & & $\mathrm{n}$ & $* * *$ \\
\hline S1137 & $19232+4422$ & 960324 & $(12.8)$ & 6.25 & 5.40 & 5.16 & 0.965 & 0.244 & / & / & & & $* * *$ \\
\hline S1143 & $19335+0358$ & 960318 & $10.4 \mathrm{v}$ & 4.99 & 4.18 & 3.72 & 2.498 & 0.619 & / & / & & & $* * *$ \\
\hline S1144 & $19339-1128$ & 971023 & $(10.6)$ & 4.93 & 4.03 & 3.91 & 1.602 & 0.450 & / & / & & $\mathrm{n}$ & $* * *$ \\
\hline S1167 & $19497+4327$ & 960324 & 10.1 & 4.19 & 3.37 & 3.12 & 3.421 & 0.963 & / & / & & $\mathrm{n}$ & $* * *$ \\
\hline $\mathrm{S} 1169$ & $19518-0803$ & 971023 & $(11.1)$ & 5.29 & 4.53 & 4.10 & 1.051 & 0.210 & / & / & & $\mathrm{n} ?$ & $* * *$ \\
\hline S1179 & $19573+4826$ & 960324 & 9.7 & 5.71 & 5.00 & 4.68 & 0.732 & 0.162 & / & / & & $\mathrm{n}$ & $* * *$ \\
\hline $\mathrm{S} 1183$ & $20004+1026$ & 971023 & 9.5 & 5.63 & 5.22 & 5.06 & 0.395 & / & / & / & & $\mathrm{n}:$ & $* * *$ \\
\hline S1186 & $20019+1518$ & 971023 & 10.7 & 5.73 & 5.32 & 5.23 & 0.371 & / & / & / & & $\mathrm{n}:$ & $* * *$ \\
\hline S1190 & $20051-0144$ & 971023 & $(11.0)$ & 3.97 & 3.28 & 2.89 & 3.844 & 1.142 & / & / & & $\mathrm{n}$ & $* * \mathrm{~K}$ \\
\hline S1211 & $20213+0047$ & 971023 & (10.9) & 2.41 & 1.64 & 1.31 & 21.92 & 7.133 & 1.2 & / & $\mathrm{S}(17)$ & $\mathrm{n} ?$ & vvv \\
\hline S1219 & $20252+3623$ & 971023 & 10.4 & 2.48 & 1.82 & 1.54 & 12.80 & 3.947 & / & / & $\mathrm{S}(16)$ & $\mathrm{n} ?$ & $* * \mathrm{~K}$ \\
\hline $\mathrm{S} 1225$ & $20305-1546$ & 971023 & $(10.5)$ & 5.15 & 4.43 & 4.29 & 0.931 & / & / & / & & $\mathrm{n}:$ & $* * *$ \\
\hline $\mathrm{S} 1227$ & $20304+2430$ & 971023 & 10.2 & 5.68 & 5.49 & 5.23 & 0.307 & / & / & / & & $\mathrm{n}:$ & $* * *$ \\
\hline $\mathrm{S} 1232$ & $20369+3742$ & 971023 & 13.1 & 3.57 & 2.88 & 2.20 & 10.36 & 2.444 & / & / & $\mathrm{S}$ & & $* * \mathrm{~K}$ \\
\hline $\mathrm{S} 1235$ & $20403+0830$ & 971023 & $10.3 \mathrm{v}$ & 5.46 & 5.12 & 4.93 & 0.618 & / & / & / & & $\mathrm{n}:$ & $* * *$ \\
\hline $\mathrm{S} 1239$ & $20455+3442$ & 971023 & 10.4 & 5.21 & 4.86 & 4.73 & 0.425 & / & / & / & & $\mathrm{n}:$ & $* * *$ \\
\hline $\mathrm{S} 1242$ & $20488+2318$ & 971021 & 9.8 & 5.98 & 5.14 & 4.98 & 0.481 & / & / & / & & $\mathrm{n}:$ & $* * *$ \\
\hline $\mathrm{S} 1248$ & $20506+3605$ & 971021 & 9.8 & 5.49 & 4.89 & 4.77 & 0.522 & / & / & / & & $\mathrm{n}:$ & $* * *$ \\
\hline S1249 & $20512+5613$ & 971021 & 10.8 & 6.04 & 5.43 & 5.25 & 0.361 & / & / & / & & $\mathrm{n}:$ & $* * *$ \\
\hline $\mathrm{S} 1253$ & $20550+2908$ & 971021 & 9.9 & 4.41 & 3.59 & 3.26 & 2.898 & 0.824 & / & / & & $\mathrm{n}$ & $* * *$ \\
\hline S1257 & $20596+6136$ & 971021 & 10.8 & 5.99 & 5.33 & 5.13 & 0.426 & / & / & / & & $\mathrm{n}:$ & $* * *$ \\
\hline $\mathrm{S} 1267$ & $21135+3133$ & 971021 & 9.2 & 5.57 & 5.08 & 4.95 & 0.523 & / & / & / & & $\mathrm{n}:$ & $* * *$ \\
\hline S1274 & $21281+5103$ & 971021 & 9.9 & 4.80 & 4.86 & 4.65 & 0.375 & / & / & / & & $\mathrm{n}:$ & $* * *$ \\
\hline $\mathrm{S} 1277$ & $21330+4037$ & 971023 & $(11.7)$ & 6.62 & 6.37 & 6.04 & 0.354 & / & / & / & & & $* * *$ \\
\hline
\end{tabular}

diagram; "n:" states that the judgment for Tc-deficiency is made only by the $K-[12]$ histogram due to the lack of the flux at $25 \mu \mathrm{m}$; and "n?" marks a star that has the proper $K-[12]$ color as a Tc-deficient $\mathrm{S}$ star but does not lie in the area populated by Tc-deficient stars in the $(K-[12])-([12]-[25])$ plot and should not be taken as a good candidate of Tc-deficient $\mathrm{S}$ stars. 
Table 3. continued.

\begin{tabular}{|c|c|c|c|c|c|c|c|c|c|c|c|c|c|}
\hline Name & IRAS & Date & $V(B)$ & $J$ & $H$ & $K$ & $F_{12}$ & $F_{25}$ & $F_{60}$ & $F_{100}$ & LRS & $\mathrm{Tc}$ & $\mathrm{CIO}$ \\
\hline S1279 & $21345+4709$ & 971021 & 10.9 & 4.73 & 4.01 & 3.70 & 1.758 & 0.485 & l & I & & $\mathrm{n}$ & $* * *$ \\
\hline S1281 & $21371+5153$ & 971021 & 10.9 & 4.76 & 4.67 & 4.69 & 0.488 & / & / & / & & $\mathrm{n}:$ & $* * *$ \\
\hline S1285 & $21512+4602$ & 971023 & 10.6 & 5.92 & 5.46 & 5.25 & 0.398 & / & / & / & & $\mathrm{n}:$ & $* * *$ \\
\hline S1286 & $21540+4806$ & 971023 & 9.0 & 4.69 & 4.33 & 3.46 & 5.949 & 1.648 & / & / & & & $* * \mathrm{v}$ \\
\hline S1290 & $22057+2925$ & 951225 & 10.8 & 6.82 & 5.92 & 5.67 & 0.324 & / & / & / & & $\mathrm{n}:$ & $* * *$ \\
\hline S1308 & $22476+4047$ & 970102 & 8.1 & 0.23 & -.13 & -.38 & 98.73 & 32.66 & 8.389 & 6.159 & $\mathrm{~S}(16)$ & $\mathrm{n} ?$ & $* * \mathrm{~K}$ \\
\hline S1309 & $22479+1737$ & 970102 & 10.0 & 3.62 & 3.38 & 3.01 & 4.594 & 1.297 & / & / & & $\mathrm{n}$ & vHK \\
\hline S1311 & $22489+0326$ & 951225 & 9.0 & 6.58 & 5.82 & 5.80 & 0.410 & / & / & / & & & $* * *$ \\
\hline S1313 & $22504+5009$ & 951225 & 9.8 & 6.44 & 5.56 & 5.27 & 0.381 & / & / & / & & $\mathrm{n}:$ & $* * *$ \\
\hline S1317 & $23029+4924$ & 951227 & 10.3 & 6.08 & 5.33 & 5.19 & 0.546 & / & / & / & & $\mathrm{n}:$ & $* * *$ \\
\hline S1319 & $23044+0200$ & 951226 & $(10.9)$ & 6.23 & 5.37 & 5.36 & 0.492 & / & / & / & & $\mathrm{n}:$ & $* * *$ \\
\hline S1326 & $23125+4921$ & 951227 & 10.7 & 5.87 & 5.07 & 4.85 & 0.602 & / & / & / & & $\mathrm{n}:$ & $* * *$ \\
\hline S1327 & $23130+5002$ & 951227 & 10.4 & 5.87 & 5.10 & 4.96 & 0.588 & / & / & / & & $\mathrm{n}:$ & $* * *$ \\
\hline S1330 & $23172+5745$ & 951225 & 11.1 & 5.22 & 4.55 & 4.42 & 0.978 & 0.222 & / & / & & $\mathrm{n}$ & $* * *$ \\
\hline S1335 & $23285+4259$ & 970102 & 9.2 & 3.81 & 3.59 & 3.37 & 3.881 & 1.094 & / & / & & & $* * *$ \\
\hline S1341 & $23466+2621$ & 970102 & 11.3 & 4.10 & 3.83 & 3.62 & 2.200 & 0.607 & / & / & & $\mathrm{n}$ & $* * *$ \\
\hline Sf9 & $02176+6110$ & 971023 & $11.2^{\mathrm{a}}$ & 4.58 & 4.49 & 4.08 & 1.398 & / & / & / & & $\mathrm{n}:$ & $* * *$ \\
\hline Sf15 & $04118+4851$ & 971023 & $11.9^{\mathrm{a}}$ & 4.65 & 4.86 & 4.79 & 0.533 & / & / & / & & $\mathrm{n}:$ & $* * *$ \\
\hline Sf57 & $20255+3758$ & 971024 & $11.4^{\mathrm{a}}$ & 4.66 & 4.73 & 4.65 & 0.670 & / & / & / & & $\mathrm{n}:$ & $* * *$ \\
\hline Sf61 & $20502+4833$ & 971024 & $11.9^{\mathrm{a}}$ & 5.36 & 5.59 & 5.40 & 0.839 & 0.187 & / & / & & & $* * *$ \\
\hline Sf68 & $21336+5343$ & 971024 & $10.2^{\mathrm{a}}$ & 4.90 & 4.95 & 4.69 & 0.389 & 1 & / & / & & $\mathrm{n}:$ & $* * *$ \\
\hline
\end{tabular}

a The $I$ magnitude is listed.

Several points in Tables 2 and 3 are worth noticing. There are 10 stars in our sample having been observed in two seasons and the magnitude differences between two observations are less than 0.1 or 0.2 . The only exception is for the Tc-deficient star S87 $=\mathrm{BD}+24^{\circ} 620=\mathrm{V} 1135$ Tau, which fainted from Dec. 1995 to March 1996 by $0.7,0$ m. 4 and $0^{\mathrm{m}} 1$ in $J, H$ and $K$ respectively. Although it became reasonably redder while getting fainter, as an AGB star usually does, the variation of 0.7 in $J$ within 80 days merits our attention in the future ${ }^{1}$. Of the Tc-rich and Tc-deficient stars studied here, 12 were observed at JHK bands in Paper I as well. Among them, half varied very little (less than $0 .{ }^{\mathrm{m}} 1$ ) compared with the previous data; some others showed somewhat large and reasonable variations $\left(0{ }^{\mathrm{m}} 2-0 . \mathrm{m} 4\right)$; but the Tc-rich star S49 $=\mathrm{HD} 14028=\mathrm{W}$ And apparently brightened by $0.9,0.8$ and 0.7 magnitude in $J$, $H$ and $K$, respectively, perhaps which is understandable as it is a well-known Mira variable with a period of 397 days and it has a very large variation range of 6.7-14.6 in $V$ magnitude (Kholopov et al. 1985-87, the General Catalog of Variable Stars, hereafter GCVS). Furthermore, it should be noted that there are $116 \mathrm{~S}$ stars studied in this paper without $J H K$ data in CIO and 21 other stars with only $K$ data in that catalog, so it appears that we are presenting the first observations in the near infrared for such a large number of S stars.

\footnotetext{
1 Dr. A. Jorissen has informed us that given the orbital elements of Jorissen et al. (1998) and an unpublished time of periastron passage $T_{0}=$ JD 2448293, the fading was, as it happened, close to the time of passage of the companion behind the giant star (Jorissen 2001). With only one measurement available, it is however impossible to tell whether this strong light change is related or not to the orbital phase.
}

\section{Discussion}

\section{1. $(K-[12])-([12]-[25])$ two-color diagram}

In Paper I, a variety of infrared two-color diagrams, including the $(J-H)-(H-K)$, the $([12]-[25])-([25]-[60])$, the $(K-[12])-([12]-[25])$ and the $(J-K)-([12]-[25])$, were presented to test the best way to distinguish those two categories of S stars through their infrared colors. We found from these diagrams that (1) both categories of S stars have almost the same color distribution in the near infrared, so they cannot be segregated by their near infrared colors; and (2) great differences occur in the 2$25 \mu \mathrm{m}$ bands: the $K-[12]$ and [12] $-[25]$ are the most sensitive colors and the $(K-[12])-([12]-[25])$ the most appropriate color-color diagram for segregation of the two kinds of stars.

Merging the data here with those in Paper I, we have near infrared magnitudes together with IRAS fluxes for a total of 35 Tc-rich S stars and 34 Tc-deficient ones, which are almost all of the $\mathrm{S}$ stars whose Tc properties are definitely known at present and that can be observed using the 1.26-m IR telescope of the Beijing Astronomical Observatory. Thus a new $(K-[12])-([12]-[25])$ diagram with a more comprehensive sample is plotted in Fig. 2 (the IRAS fluxes are transformed into magnitudes without color corrections according to Cheeseman et al. 1989), which shows no fundamental difference with the old one in Paper I. Now the Tc-deficient stars are actually concentrated on the area of $0.3<K-[12]<1.0$ and $-0.1<[12]-[25]<0.25$, which would be taken as the criteria of a candidate belonging to the Tc-deficient family. 


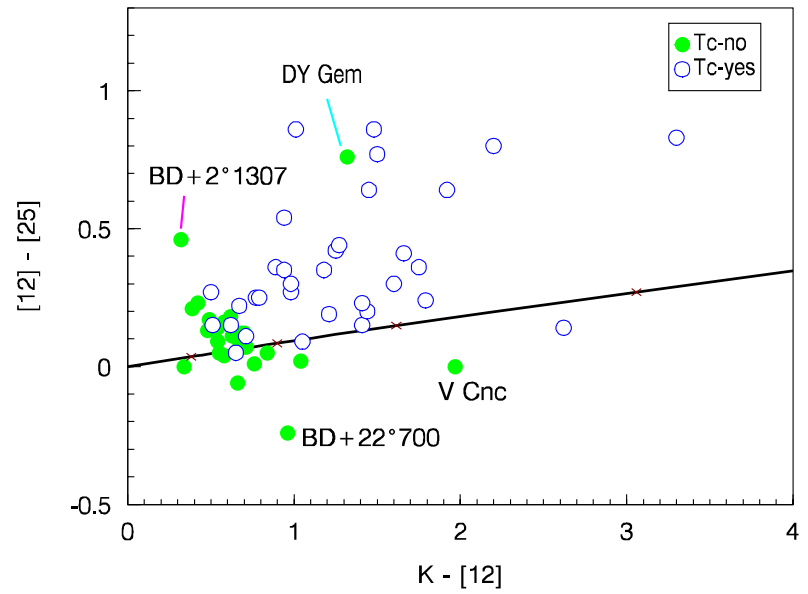

Fig. 2. $(K-[12])-([12]-[25])$ diagram for Tc-rich and Tcdeficient $\mathrm{S}$ stars, where the open circles stand for Tc-rich stars and the filled circles for Tc-deficient stars. The solid line represents black body colors, with crosses (from left to right) corresponding to temperatures of 5000, 3000, 2000 and $1250 \mathrm{~K}$ respectively.

Tc-deficient $\mathrm{S}$ stars $\mathrm{S} 96=\mathrm{BD}+22^{\circ} 700, \mathrm{~S} 231=$ DY Gem, S233 $=\mathrm{BD}+2^{\circ} 1307$ and $\mathrm{S} 494=\mathrm{V}$ Cnc, and Tc-rich ones S114 = o ${ }^{1}$ Ori, S422 = NQ Pup, S1053 = HD 170970 and $\mathrm{S} 1070=\mathrm{V} 679$ Oph are exceptions to such a partition, as previously noted and explained by Groenewegen (1993), Jorissen et al. (1993), Chen et al. (Paper I) and Jorissen \& Knapp (1998). An additional Tc-rich star, $\mathrm{S} 1193=\mathrm{BD}+4^{\circ} 4354$, is located on the boundary of the Tc-deficient region (with $K-[12]=0.67$ and $[12]-[25]=0.22)$. A radial-velocity monitoring for it over 3000 days showed no evidence of binarity (Jorissen et al. 1998). We also note that despite being claimed with quality 3 in IRAS PSC, the $25 \mu \mathrm{m}$ flux of BD $+4^{\circ} 4354$ is relatively weak (close to the IRAS detection threshold) and is associated with a relatively large uncertainty of $15 \%$, which we would rather propose to be responsible for the improperly bluer [12] - [25] color. Moreover, it is interesting to note that Tc-deficient Mira star V Cnc varies in $V$ magnitude between 7.5 and 13.9 (GCVS) but, surprisingly, has hardly varied in $J, H$ and $K$ from the measurement of Catchpole et al. (1979) to that of Chen et al. (1988), then to that in Paper I, and then to that given here. Further investigation for $\mathrm{V}$ Cnc is needed.

Following the paradigm of Fig. 2, the $(K-[12])-([12]-$ $[25])$ two-color diagram for the candidates is plotted in Fig. 3 by which, from all 51 candidates with good quality $25 \mu \mathrm{m}$ fluxes, we may extract 39 that should, with high probability, be Tc-deficient according to the infrared criteria mentioned above. The results are presented in Table 3 with a letter " $\mathrm{n}$ " in the column labelled "Tc".

\section{2. $K-[12]$ histogram}

As mentioned above, only 51 stars, out of the 124 candidates studied here, have good fluxes in $25 \mu \mathrm{m}$ band (see Table 3 ). For the remainder, $K-[12]$ is the only infrared color that can be utilized to classify them. Happily, the

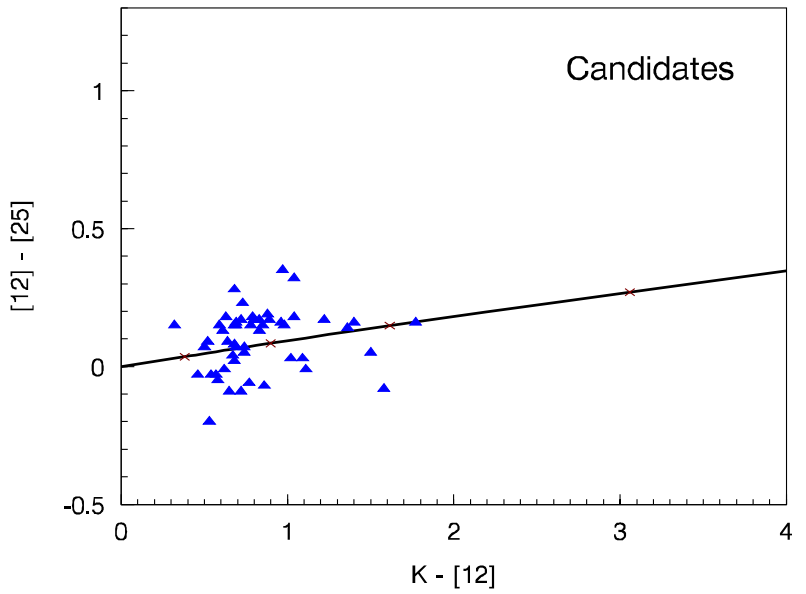

Fig. 3. $(K-[12])-([12]-[25])$ diagram for the observed candidates. Crosses on the black body line indicate the same temperatures as in Fig. 2.

$K-[12]$ appears a more efficient dividing tool than the [12] - [25]: most of the stars distinguishable in the twocolor diagram are in fact well discernible even if one uses the $K-[12]$ color alone (as may be seen from Fig. 2).

The histogram of $K-[12]$ color for Tc-rich and Tcdeficient $\mathrm{S}$ stars observed here and in Paper I is drawn with a sample size of 0.2 in Fig. 4, which includes more stars than Fig. 2. This histogram makes it immediately obvious that there is a criterion line at $K-[12]=1.0$. Almost all Tc-deficient stars can be found in the left area $(K-[12]<$ 1.0). The two peculiar objects with $K-[12]$ apparently larger than 1 are S231 = DY Gem and S494 = V Cnc, which have been mentioned in Sect. 4.1. In addition three stars have $K-[12] \sim 1$ (actually, in a range 0.9-1.1). On the other hand, most, though not all, Tc-rich ones are distributed in the right area (with $K-[12]>1.0$ ). Besides the five Tc-rich stars for which both the $K-[12]$ and $[12]-[25]$ colors look abnormal, as shown in Fig. 2, four others, $\mathrm{S} 803=\mathrm{S}$ UMa (out of the region populated by Tc-deficient $\mathrm{S}$ stars in Fig. 2 ) and $\mathrm{S} 89=\mathrm{BD}+23^{\circ} 654$, $\mathrm{S} 411=\mathrm{HD} 63733$ and $\mathrm{S} 416=\mathrm{BD}-4^{\circ} 2121$ (these three stars are out of but close to the Tc-deficient region in Fig. 2), are also located to the left in Fig. 4. The ambiguity for HD 63733 has been commented on by Jorissen et al. (1993), and a spectroscopic orbit with a period of $1160 \mathrm{~d}$ has been derived for it by Udry et al. (1998). The radialvelocity data presented by Udry et al. (1998) also revealed clear velocity variation associated with orbital motion for the Mira star S UMa, but no such variation was seen (also see Jorissen et al. 1998) for $\mathrm{BD}-4^{\circ} 2121$.

From the $K-[12]$ histogram for the candidates presented in Fig. 5 it is seen that an overwhelming majority of the candidates have $K-[12]<1.0$ and thus likely belong to the Tc-deficient category. This conclusion implies that our preliminary selection criteria proposed in Sect. 2 stand to reason. The possible Tc-deficient stars judged only by $K-[12]$ color (due to the absence of a good $25 \mu \mathrm{m}$ flux) are indicated with "n:" in the "Tc" column of Table 3 . We take these stars as very likely Tc-deficient ones not only 


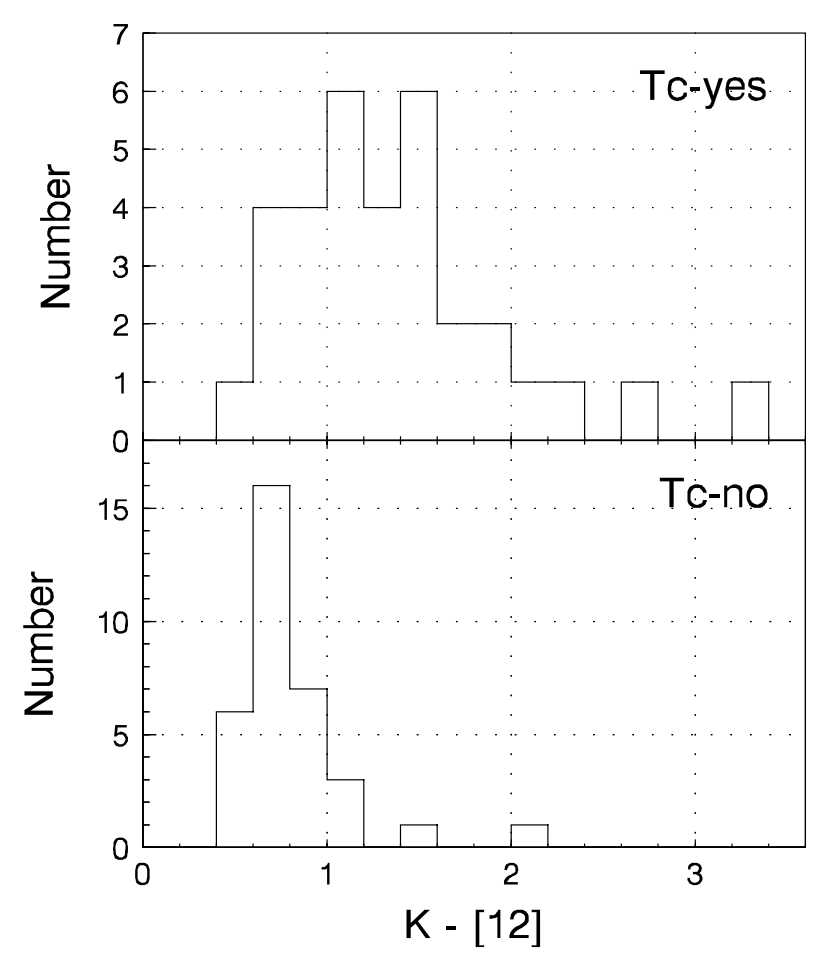

Fig. 4. $K-[12]$ histogram for Tc-rich and Tc-deficient S stars.

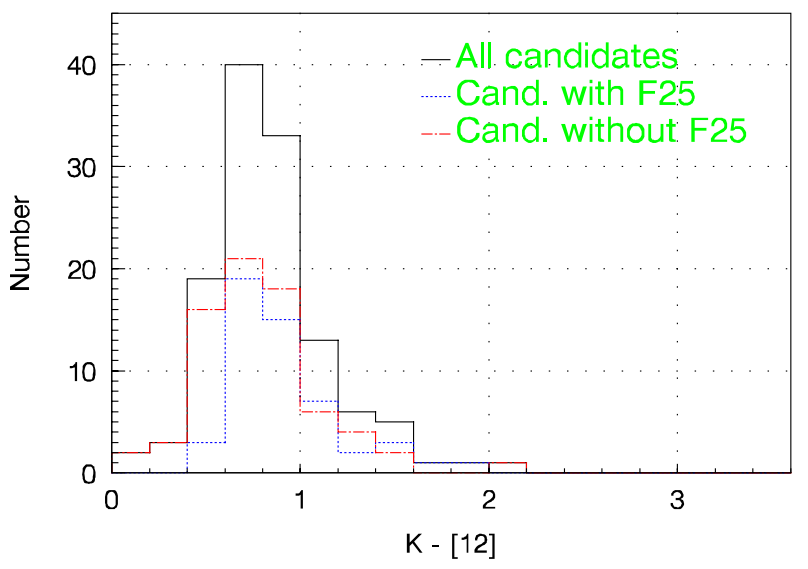

Fig. 5. $K-[12]$ histogram for the observed candidates (solid line). The distributions of the candidates with (dotted histogram) and without (dot-dashed histogram) good quality $25 \mu \mathrm{m}$ fluxes are also presented.

because the $K-[12]$ color is a relatively reliable rule for the distinction between the two categories as pointed out above, but also because the absence of good $25 \mu \mathrm{m}$ flux itself implies quite a low flux near or under the IRAS detection threshold, which leads to a relatively small [12] - [25] color that should characterize a Tc-deficient star. Of the 73 candidates without good fluxes in the $25 \mu \mathrm{m}$ band, 65 are members of the Tc-deficient class (as indicated by the dot-dashed histogram in Fig. 5). The very high frequency $(65 / 73=89 \%)$ itself supports the supposition that such candidates frequently show Tc-deficient properties.

Some other stars have $K-[12]<1.0$ but do not fall into the Tc-deficient area of $(K-[12])-([12]-[25])$ diagram due to improper [12] - [25] colors; therefore they
Table 4. Distributions of Tc-rich and Tc-deficient S stars among the LRS classes.

\begin{tabular}{llllll}
\hline & Sum & E & F & S & I \\
\hline Tc-no & 11 & $\ldots$ & $2(18 \%)$ & $8(73 \%)$ & $1(9 \%)$ \\
Tc-yes & 30 & $9(30 \%)$ & $8(27 \%)$ & $11(37 \%)$ & $2(7 \%)$ \\
\hline
\end{tabular}

should not be looked upon as good candidate Tc-deficient S stars and they are indicated with "n?" in Table 3. Four stars, S1169, S1211, S1219 and S1308, are such cases.

\subsection{LRS classification and spectral energy distribution}

Chen \& Kwok (1993) thoroughly studied the IRAS lowresolution spectra of $\mathrm{S}$ stars and demonstrated the great importance of the LRS (especially the LRS letter class) to the understanding of the circumstellar properties of this species of chemically peculiar red giants. Jorissen et al. (1993) and Jorissen \& Knapp (1998) further discussed the distribution of the various LRS classes in partitioned infrared two-color diagrams. In addition, the different shapes of the LRS spectra of the two kinds of $\mathrm{S}$ stars were showed by Groenewegen (1993). A comparison of the LRS letter classification between the two kinds was made by Chen \& Kwok (1993) and in Paper I. Now, we await higher-quality ISO data to bring us new information concerning the infrared spectra of S stars. However it seems that the older IRAS LRS material is still of interest because the S stars with ISO observation are very limited in number. A recent inquiry made by us reveals that there are only $22 \mathrm{~S}$ stars (among which 11 are Tc-rich, 3 Tc-deficient and 8 Tc-unknown) with ISO SWS and/or LWS spectral data.

What is presented in Table 4 is an extension of the comparisons made by Chen \& Kwok (1993) and in Paper I. The present comparison is based on a relatively comprehensive sample reassembled in Table 1. For each of the two categories of S stars the sum of the stars with specific LRS classification is given and the numbers (along with the corresponding percentages) in every LRS group are listed. There is a weak correlation between the Tc content and the LRS class: most Tc-deficient $\mathrm{S}$ stars are in LRS group $\mathrm{S}$, which is characterized by photospheric features; all $\mathrm{S}$ stars with silicate emission features (in group E) exhibit Tc in their spectra. However, it should be noted that the Tc-rich stars are distributed almost equally among groups E, F and S, and the absolute number of Tcrich group $\mathrm{S}$ stars is even more than that of Tc-deficient group S stars. Only 6 of 124 candidates have an LRS classification and all are in group S. On the other hand, two of them are identified as (quite possible) Tc-deficient members and the others not (see Table 3). This shows again that the LRS classification does not seem so efficient an index for dividing S stars into Tc-rich and Tc-deficient ones unless one can exclude a star of group E without doubt from the Tc-deficient family. 
In Paper I we have investigated some Tc-rich and Tcdeficient $\mathrm{S}$ stars in the broad band spectral energy distribution (SED) from $B$ (or $V$ ) band to $60 \mu \mathrm{m}$ or to $100 \mu \mathrm{m}$ band and found that all the SEDs of Tc-deficient stars can be fitted to a single blackbody curve which corresponds to only the photospheric property, whereas many Tc-rich stars (mainly those in LRS group E) demand double blackbody fitting (see Xiong et al. 1994 for the model) which is related to both photospheric and circumstellar properties. This finding is now verified through the SED pattern of 10 more Tc-rich or Tc-deficient sources observed in this paper with good measurements in $60 \mu \mathrm{m}$. Moreover, we have 5 candidates in Table 3 which have enough data to fit a SED. None need a double blackbody mode, as 4 stars are noted in LRS group S. Among them, S57, S176 and S212 are possibly Tc-deficient according to the color criteria, but S1211 and S1308 are not (for a somewhat larger $[12]-[25])$.

\section{Summary and remark}

Those two categories of S stars lie in different phases of stellar evolution. The contamination of Tc-deficient extrinsic $\mathrm{S}$ stars on Tc-rich intrinsic $\mathrm{S}$ stars (real $S$ stars at $A G B$ phase) has thus considerably affected our knowledge of AGB stars. Therefore it is greatly meaningful to separate the Tc-deficient kind from the Tc-rich one.

In this paper we present near infrared photometry of $161 \mathrm{~S}$ stars (for most of them, these are the first observations in the near infrared). The $(K-[12])-([12]-[25])$ two-color diagram and the $K-[12]$ histogram are presented on an extended sample to further investigate the distinguishable properties of the two $\mathrm{S}$ star families. Then a larger number of candidates are tested in the infrared property and 104 stars are identified as very likely Tcdeficient $\mathrm{S}$ stars. Moreover the IRAS LRS classification and the spectral energy distribution are discussed for those known Tc-rich and Tc-deficient S stars, and for the candidates as well. These infrared-selected Tc-deficient S stars make up a good sample for a deep study of the physical properties and the evolutionary status of this species of S stars.

Acknowledgements. We are very grateful to the referee Dr. A. Jorissen for his helpful suggestions. We also thank the staff of Xinglong Station, Joint Lab. for Optical Astronomy, CAS, especially Ms. F.-y. Li, Mr. Z.-j. Zhao and Mr. Z.-g. Hou, for their assistant observation. This work is supported by the National Natural Science Foundation of China and the Chinese Academy of Sciences.

\section{References}

Brown, J. A., Smith, V. V., Lambert, D. L., et al. 1990, AJ, 99, 1930

Carquillat, J. M., Jorissen, A., Udry, S., \& Ginestet, N. 1998, A\&AS, 131, 49

Catchpole, R. M., Robertson, B. S. C., Lloyd Evans, T. H. H., et al. 1979, SAAO Circ., 1, 61
Cheeseman, P., Stutz, J., Self, M., et al. 1989, NASA RP-1217, 9

Chen, P. S., Gao, H., Chen, Y. K., \& Dong, H. W. 1988, A\&AS, 72, 239

Chen, P. S., \& Kwok, S. 1993, ApJ, 416, 769

Chen, P. S., Gao, H., \& Jorissen, A. 1995, A\&AS, 113, 51 (CGJ)

Chen, P.-S., Wang X.-H., \& Xiong, G.-Z. 1998, A\&A, 333, 613 (Paper I)

Feast, M. W., Catchpole, R. M., \& Glass, I. S. 1976, MNRAS, $174,81 p$

Gezari, D. Y., Schmitz, M., Pitts, P. S., \& Mead, J. M. 1993, NASA RP-1294

Groenewegen, M. A. T. 1993, A\&A, 271, 180

HST Guide Star Catalog 1992, CD-ROM, Version 1.1 (STScI, Baltimore)

Iben, I., \& Renzini, A. 1983, ARA\&A, 21, 271

Johnson, H. R., Ake, T. B., Ameen, M. M., \& Brown, J. A. 1990, in Miras to Planetary Nebulae: Which Path for Stellar Evolution?, ed. M. O. Mennessier, \& A. Omont (Éditions Frontières, Gif-sur-Yvette, France), 332

Johnson, H. R., Ake, T. B., \& Ameen, M. M. 1993, ApJ, 402, 667

Joint IRAS Science Working Group 1986, IRAS Catalogs and Atlases, Atlas of Low-Resolution Spectra (LRS), A\&AS, 65,607

Joint IRAS Science Working Group 1988, IRAS Catalogs and Atlases, Explanatory Supplement (GPO, Washington DC)

Joint IRAS Science Working Group 1988, IRAS Point Source Catalog, Version 2 (GPO, Washington DC) (PSC)

Jorissen, A. 2001, private communication

Jorissen, A., \& Knapp, G. R. 1998, A\&AS, 129, 363

Jorissen, A., \& Mayor, M. 1988, A\&A, 198, 187

Jorissen, A., \& Mayor, M. 1992, A\&A, 260, 115

Jorissen, A., Frayer, D. T., Johnson, H. R., Mayor, M., \& Smith, V. V. 1993, A\&A, 271, 463

Jorissen, A., Van Eck, S., Mayor, M., \& Udry, S. 1998, A\&A, 332,877

Jura, M. 1986, Ir. Astron. J., 17, 322

Kholopov, P. N., Samus, N. N., Frolov, M. S., et al. 1985-1987, General Catalog of Variable Stars, 4th edition (Nauka, Moscow) (GCVS)

Kwok, S., Volk, K., \& Bidelman, W. P. 1997, ApJS, 112, 557 (LRSLC)

Little, S. J., Little-Marenin, I. R., \& Hagen-Bauer, W. 1987, AJ, 94, 981

Little-Marenin, I. R., \& Little, S. J. 1979, AJ, 84, 1374

Rieke, G. H., \& Lebofsky, M. J. 1985, ApJ, 288, 618

Scalo, J. M., \& Miller, G. E. 1981, ApJ, 246, 251

Smith, V. V., \& Lambert, D. L. 1988, ApJ, 333, 219

Smith, V. V., \& Lambert, D. L. 1990, ApJS, 72, 387

Stephenson, C. B. 1984, General Catalogue of Galactic S Stars, Pub. Warner \& Swasey Obs., 3, 1 (GCGSS)

Stephenson, C. B. 1990, AJ, 100, 569

Udry, S., Jorissen, A., Mayor, M., \& van Eck, S. 1998, A\&AS, 131, 25

Van Eck, S., \& Jorissen, A. 1999, A\&A, 345, 127

Van Eck, S., \& Jorissen, A. 2000, A\&A, 360, 196

Van Eck, S., Jorissen, A., Udry, S., Mayor, M., \& Pernier, B. 1998, A\&A, 329, 971

van Herk, G. 1965, Bull. Astron. Inst. Neth., 18, 71

Volk, K., \& Cohen, M. 1989, AJ, 98, 931

Xiong, G. Z., Chen, P. S., \& Chen, J. B. 1994, Acta Astron. Sin., 35,20 